\title{
An Enhanced Collaborative Optimization Approach with Design Structure Matrix Algorithms to Group and Decouple Multidisciplines
}

\author{
Xifan Yao, ${ }^{1,2}$ Jifeng Zhou, ${ }^{2}$ Yongxiang $\mathrm{Li}^{2}{ }^{2}$ and Erhui $\mathrm{Liu}^{2}$ \\ ${ }^{1}$ State Key Laboratory of Automotive Simulation and Control, Jilin University, Changchun 130025, China \\ ${ }^{2}$ School of Mechanical \& Automotive Engineering, South China University of Technology, Guangzhou 510640, China \\ Correspondence should be addressed to Xifan Yao; mexfyao@scut.edu.cn
}

Received 7 April 2016; Accepted 12 July 2016

Academic Editor: Ruben Specogna

Copyright (c) 2016 Xifan Yao et al. This is an open access article distributed under the Creative Commons Attribution License, which permits unrestricted use, distribution, and reproduction in any medium, provided the original work is properly cited.

\begin{abstract}
To improve the solution efficiency and reliability of multidisciplinary design optimization (MDO), an enhanced MDO approach, called sequenced collaborative optimization (SCO), is proposed. The proposed approach introduces the design structure matrix (DSM) to describe the coupling effects among disciplines and aggregates those mutually coupling disciplines into the strong tie groups among similar ones and the weak tie among heterogeneous ones through clustering algorithms. Further, those in the same group are sequenced by the DSM division algorithm. Moreover, by adding constraints, the groups are made independent, resulting in a tree structure without loops, thus decoupling the original multidisciplinary problem into several independent collaborative optimization modules. In the end, an example is employed to verify the efficiency and reliability of the approach.
\end{abstract}

\section{Introduction}

Multidisciplinary design optimization (MDO) is a kind of methodology to design complex systems and subsystems by fully exploring and utilizing the interaction mechanism of the systems. Logically, a complex engineering system can usually be divided into multiple disciplines, each of which is an abstract form of one or several fields. In the process of system level optimization design, the coupling effect among various disciplines should be taken into account, thus to realize the optimization solution [1]. In designing complex products, the application of MDO greatly improves the performance of complex products, shortens the design cycle, and is of great significance.

The essence of MDO is reflected in using knowledge from multiple disciplines to integrate models and analysis tools, organizing and managing the designing process through the effective design and optimization strategy and making full use of the coordination effect caused by the interaction among various disciplines to obtain the overall optimal solution [2]. Optimization strategy not only affects the results of MDO but also plays a crucial role in the optimization efficiency $[3,4]$. With the improvement of MDO, researches mainly focus on its application involved with complicatedly designed objects and corresponding MDO platforms [5]. Quantities of MDO platforms, such as IHAT (Integrated Hypersonic Aeromechanics Tool), MDOPT (multidisciplinary design optimization), IDEA (Integrated Design and Engineering Analysis) [6], OpenMDAO [7], and Isight [8], simplify MDO design process and enhance its efficiency. There are also many researches on the applications of MDO. For example, Meruva et al. [9] applied multidisciplinary approaches in predicting the wear and fatigue life for the optimization of lead screw actuators; Cheng et al. [10] presented MDO method for a hydrostatic rotary table based on sensitivity analysis, which was used to achieve an optimal design with less cost and better performance; Su et al. [11] applied MDO technology into the design of an integral bus body structure to improve its synthetic performance, considering lightweight, stiffness, strength, vibration mode, and rollover. The research results of the optimization platforms and application technologies mentioned above are of significance in designing complex 


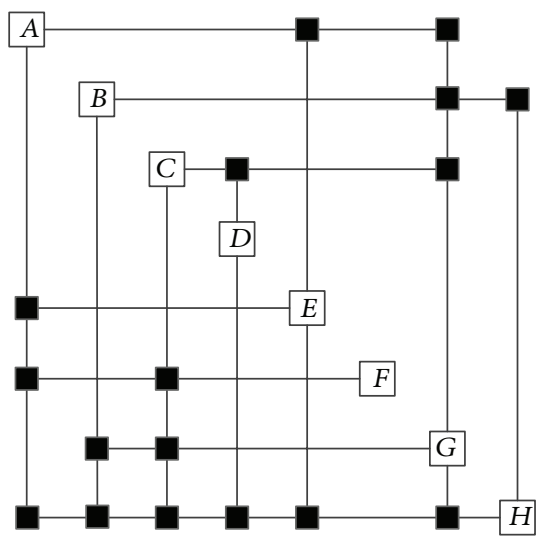

(a)

\begin{tabular}{lcccccccc}
\hline Discipline & $A$ & $B$ & $C$ & $D$ & $E$ & $F$ & $G$ & $H$ \\
\hline$A$ & $A$ & 0 & 0 & 0 & 1 & 0 & 1 & 0 \\
$B$ & 0 & $B$ & 0 & 0 & 0 & 0 & 1 & 1 \\
$C$ & 0 & 0 & $C$ & 1 & 0 & 0 & 1 & 0 \\
$D$ & 0 & 0 & 0 & $D$ & 0 & 0 & 0 & 0 \\
$E$ & 1 & 0 & 0 & 0 & $E$ & 0 & 0 & 0 \\
$F$ & 1 & 0 & 1 & 0 & 0 & $F$ & 0 & 0 \\
$G$ & 0 & 1 & 1 & 0 & 0 & 0 & $G$ & 0 \\
$H$ & 1 & 1 & 1 & 1 & 1 & 0 & 1 & $H$ \\
\hline
\end{tabular}

(b)

FIGURE 1: DSM for representing disciplines.

products. However, due to complex information interaction and coupling among various disciplines, the application of MDO faces the obstacles of complicated coordination process and heavy computation cost [12].

Therefore, this paper proposes a so-called sequenced collaborative optimization (SCO) approach, where design structure matrix (DSM) is applied into the solving process of MDO and is used to describe links among various disciplines. Through clustering algorithms, the multiple disciplines are divided into different groups with strong ties in the same group and weak ties in different ones. The disciplines in the same group are sequenced by the division algorithm, while the groups are made independent by adding some constraints. As such, tree structures without loop are obtained so as to avoid low efficiency and nested loops that are difficult to converge and facilitate the practical application of MDO technology.

\section{DSM Modeling and Analysis of Discipline Interaction}

DSM is a matrix tool for the planning and analysis of product design process developed by Dr. Steward [13]. The design of modern products is increasingly integrating knowledge, information, and technology and its objects are also becoming more and more complicated. For example, the design of large complex products, such as spacecraft, aircraft, automobiles, and ships, is a multidisciplinary, complex, and highspeed iterative process. DSM can directly display the relationships between disciplines or subsystems and improves design quality and efficiency. For example, Habib [14] modeled the subsystems of mechanical and electrical products by DSM, managed information interaction between subsystems and components, and modularized design objects, which simplified the design process; Lambe and Martins [15] extended DSM, on the basis of which components were added to define iterative processes, different line types were used to display data and process connection independently, and components executed sequentially were defined by number. There are also some researches on the application process of the extended
DSM in MDO models. For example, Su et al. [5] put forward general multidisciplinary optimization framework based on a discipline relationship matrix, gave a set of normative, general, and automatic solving strategies by means of discipline input/output variables and the conversion relationship of MDO optimization model, and provided a more intuitive and convenient way for the optimization platform to solve MDO problems. The above researches explored the application of DSM into MDO issues but did not connect DSM and the solving process of MDO directly. Instead, they mainly focused on studying information management and design planning by DSM and lacked discussion on the actual solving process. In [16], fuzzy DSM was used to describe the multidisciplinary design process, which was simplified and coupled. It reduced the iteration between subjects, but the application is rather complicated when the number of subjects is relatively large. Closely connecting DSM and the solving process of MDO, this paper simplifies discipline relationships by DSM and uses concrete optimization examples to elaborate it.

2.1. Discipline Interaction Modeling. When DSM is used to represent the interaction relationships between disciplines, $n$ disciplines are first sequentially arranged, forming $n \times$ $n$ matrix, whose rows and columns correspond to disciplines with the same sequence. The units on the diagonal of the matrix represent disciplines and other elements are interaction relationships between disciplines, where 1 and 0 indicate whether there is an interaction or not, respectively. If subject $i$ needs the information output of subject $j$ as information input, then the matrix element $a_{i j}=1$ or else $a_{i j}=0$. Figure 1 shows the DSM of the interaction information of a multidisciplinary optimization problem. The correlation information is composed of eight disciplines. Each row in the figure represents the information input of the corresponding discipline, while each column stands for the information output for other disciplines. The unit information above the diagonal indicates the disciplinary feedback information, where subsequent discipline results will affect previous results. The unit information below the diagonal refers to the disciplinary feed-forward information, 
where previous results will influence subsequent results. At the time of design optimization, transformation processing of matrix should be utilized to convert the subject matrix into the lower triangular form as far as possible, so as to avoid the complex nested loop and improve optimization efficiency by solving multiple disciplines sequentially.

2.2. Clustering Analysis. Clustering algorithm can reorder disciplines in the DSM and form targeted discipline clustering modules that comprise disciplines with strong ties and meanwhile among which minimized constrained association is kept. In optimizing and reconfiguring the initial DSM of a research object, two core data points, that is bid value and coordination cost [17], are introduced. A discipline is selected arbitrarily and is grouped by calculating its bid in all groups. The bid of a discipline shows dependencies between the subject itself and other groups, which can be calculated as follows:

$$
\operatorname{Bid}_{j}=\frac{\left(\sum I_{j}\right)^{\lambda_{d}}}{\left(N_{j}\right)^{\lambda_{b}}},
$$

where $\mathrm{Bid}_{j}$ is the bid value of the $j($ th) clustering module for the selected discipline; $\sum I_{j}$ stands for the sum of the interactions among the system discipline and all disciplines in the $j\left(\right.$ th) clustering module; $N_{j}$ is the number of disciplines in the $j\left(\right.$ th) clustering module; $\lambda_{d}$ represents the interactive weight exponent; and $\lambda_{b}$ refers to the bid exponent of the clustering module.

The coordination cost is the objective function, which is calculated circularly for the minimum value to determine the final clustering scheme. It is proportional to the number of disciplines in the clustering modules and the interaction among disciplines. The coordination cost value can be divided into those within clustering modules and those outside the modules $[17,18]$, as calculated as follows:

$$
\begin{aligned}
\operatorname{Intra} C & =(\operatorname{DSM}(j, k)+\operatorname{DSM}(k, j)) * N_{y}^{\lambda_{c}}, \\
\operatorname{Extra} C & =(\operatorname{DSM}(j, k)+\operatorname{DSM}(k, j)) * N^{\lambda_{c}}, \\
\operatorname{Total} C & =\sum \operatorname{Intra} C+\sum \operatorname{Extra} C,
\end{aligned}
$$

where Intra $C$ represents the coordination cost of a particular clustering module; Extra $C$ is the clustering cost outside a particular clustering module; Total $C$ stands for the overall coordination cost of the target system; $\operatorname{DSM}(j, k)$ and $\operatorname{DSM}(k, j)$ indicate the interaction between subjects $j$ and $k$ and that between subjects $k$ and $j$, respectively; $N_{y}$ and $N$ are the number of disciplines of clustering module $Y$ and that of the target system, respectively; and $\lambda_{c}$ is the weight exponent of the clustering modules.

By a loop calculation of bid values and coordination costs, the clustering module that the target system discipline belongs to can be identified, and finally the clustering operation of the DSM of the target system can be completed. Due to its strong advantage in matrix operations, Matlab software is used for programming in this paper so as to achieve the DSM clustering algorithm of a target system. The specific

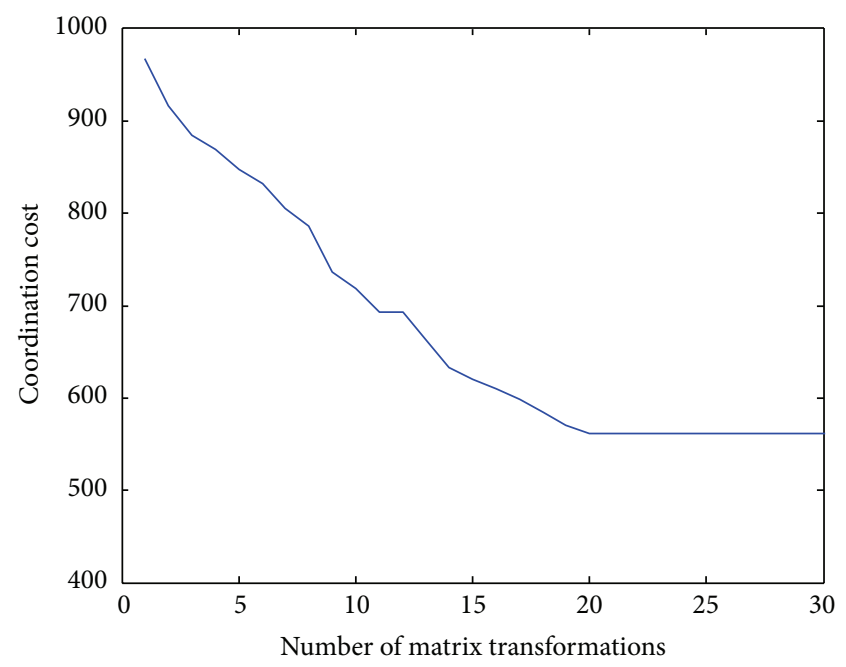

FIGURE 2: The curve of coordination cost.

procedures are as follows:

(1) Initialize the DSM, where each discipline is taken as a module.

(2) Calculate the coordination cost of the initial DSM, Total C.

(3) Arbitrarily select a discipline from the DSM matrix to form a module.

(4) Calculate the bids of all other modules relatively to the particular discipline selected.

(5) Place the selected discipline in the module which has the highest bid value.

(6) If the newly calculated Total $C$ is smaller than the previous one, the selected discipline is incorporated in the module, or else it remains independent of the module.

(7) Choose a new task to repeat Steps (3)-(6) until all tasks have been traversed and no further clustering operations can be carried out.

After the above clustering algorithm is applied to the discipline relation matrix in Figure 1, the eight subdisciplines are aggregated into two subgroups according to their interaction relationships, that is, $B, C, D, G$, and $H$ in a group and $A$, $E$, and $F$ in the other one, where in-group interactions keep close while cross-group interactions remain scarce, which is consistent with the expected result. The curve of coordination cost is shown in Figure 2, and the clustered result is shown in Figure 3.

2.3. Partitioning Operation. After clustering analysis, the discipline matrix forms multiple discipline groups, which lays a foundation for the parallel calculation of multidiscipline optimization, but the coupling relations within a discipline group is still complicated, which is not conducive to the solving process. Partitioning algorithm can rearrange the disciplines in the same group so as to achieve the purpose of reducing 


\begin{tabular}{lcccccccc}
\hline Discipline & $B$ & $C$ & $D$ & $G$ & $H$ & $A$ & $E$ & $F$ \\
\hline$B$ & $B$ & 0 & 0 & 1 & 1 & 0 & 0 & 0 \\
$C$ & 0 & $C$ & 1 & 1 & 0 & 0 & 0 & 0 \\
$D$ & 0 & 0 & $D$ & 0 & 0 & 0 & 0 & 0 \\
$G$ & 1 & 1 & 0 & $G$ & 0 & 0 & 0 & 0 \\
$H$ & 1 & 1 & 1 & 1 & $H$ & 1 & 1 & 0 \\
$A$ & 0 & 0 & 0 & 1 & 0 & $A$ & 1 & 0 \\
$E$ & 0 & 0 & 0 & 0 & 0 & 1 & $E$ & 0 \\
$F$ & 0 & 1 & 0 & 0 & 0 & 1 & 0 & $F$ \\
\hline
\end{tabular}

Figure 3: The result after clustering operation on the DSM in Figure 1.

feedback relevance; that is, try to reduce cells where there are correlations among corresponding disciplines represented on the upper triangular part of the discipline subgroup matrix and convert it into a lower triangular matrix as far as possible. The specific steps are as follows:

(1) Determine the discipline elements in the system that can perform independently without requiring the output information of other disciplines as the input information; that is, find out the row whose cell values are zero. Place such elements in the forefront of the matrix and at the meantime convert the corresponding column. Repeat the step until all the elements of zero values in the corresponding row are rearranged completely.

(2) Determine the discipline elements that do not provide information for other disciplines; that is, find out the column whose cell values are zero. As the disciplines corresponding to the column do not provide information for other disciplines, place the column as the last one of the matrix and at the same time convert the corresponding row. Repeat the step until all the elements of zero values in the corresponding column are rearranged completely.

(3) After the above two steps, if all the elements of the matrix targeted have been rearranged, partitioning operation is completed. If not, there are coupling relationships between the remaining discipline elements. Finding out the coupling disciplines through, for example, path search method, adjacency matrix method, reachable matrix method, and triangulation algorithm, is needed.

(4) Treat the coupling disciplines as a whole, and use an element symbol to represent them in the discipline matrix. Further repeat a partitioning operation on the new formed matrix until all elements complete rearrangement.

(5) Split the elements that are treated as a whole. The resulting matrix obtained is the DSM of the targeted system after being partitioned and reconstructed.

\begin{tabular}{lllllllll}
\hline Discipline & $D$ & $G$ & $B$ & $C$ & $H$ & $A$ & $E$ & $F$ \\
\hline$D$ & $D$ & 0 & 0 & 0 & 0 & 0 & 0 & 0 \\
$G$ & 0 & $G$ & 1 & 1 & 0 & 0 & 0 & 0 \\
$B$ & 0 & 1 & $B$ & 0 & 1 & 0 & 0 & 0 \\
$C$ & 1 & 1 & 0 & $C$ & 0 & 0 & 0 & 0 \\
$H$ & 1 & 1 & 1 & 1 & $H$ & 1 & 1 & 0 \\
$A$ & 0 & 1 & 0 & 0 & 0 & $A$ & 1 & 0 \\
$E$ & 0 & 0 & 0 & 0 & 0 & 1 & $E$ & 0 \\
$F$ & 0 & 0 & 0 & 1 & 0 & 1 & 0 & $F$ \\
\hline
\end{tabular}

FIGURE 4: The result after partitioning operation on the two clustered subgroups in Figure 3.

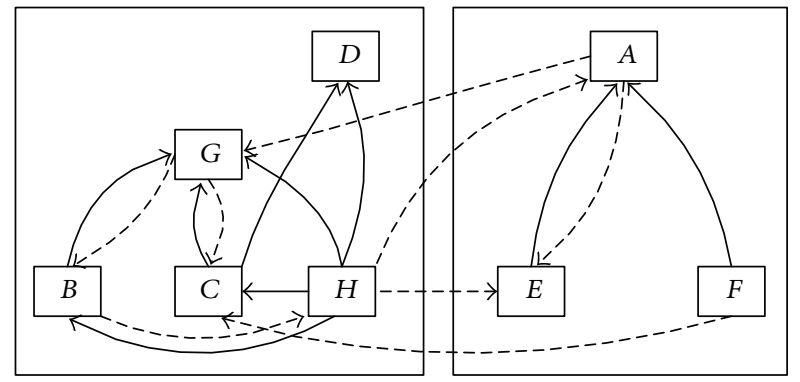

Figure 5: The directed graph for decoupling.

According to the above steps, the result as shown in Figure 4 is obtained after partitioning operation on the two clustered subgroups shown in Figure 3.

\section{Decoupling and Optimization}

With the continuous development of MDO technology, the idea of multidisciplines has gradually evolved into a modular optimal strategy. As mentioned above, through clustering operation of multidisciplinary interaction matrix, mutually coupling disciplines can be aggregated into different disciplinary groups with strong ties among similar ones and weak ties among heterogeneous ones. And the solving order of disciplines in each group can be further rearranged after division operation of each discipline group. Even so, the discipline interaction among different groups and the coupling relationships between different disciplines in the same group have not be completely eliminated. By adding the specified constraints, different disciplinary groups can be made independent of each other, and disciplines within the same group can be completely decoupled, through which the parallel computing of multidisciplinary groups can be realized. As shown in Figure 5, the decoupling directed graph is obtained after clustering and division operations. The direction of an arrow expresses the dependencies between the disciplines. For example, the arrows from discipline $G$ to disciplines $B$ and $C$ represent that the analysis of discipline $G$ depends on the outputs of disciplines $B$ and $C$, and the 


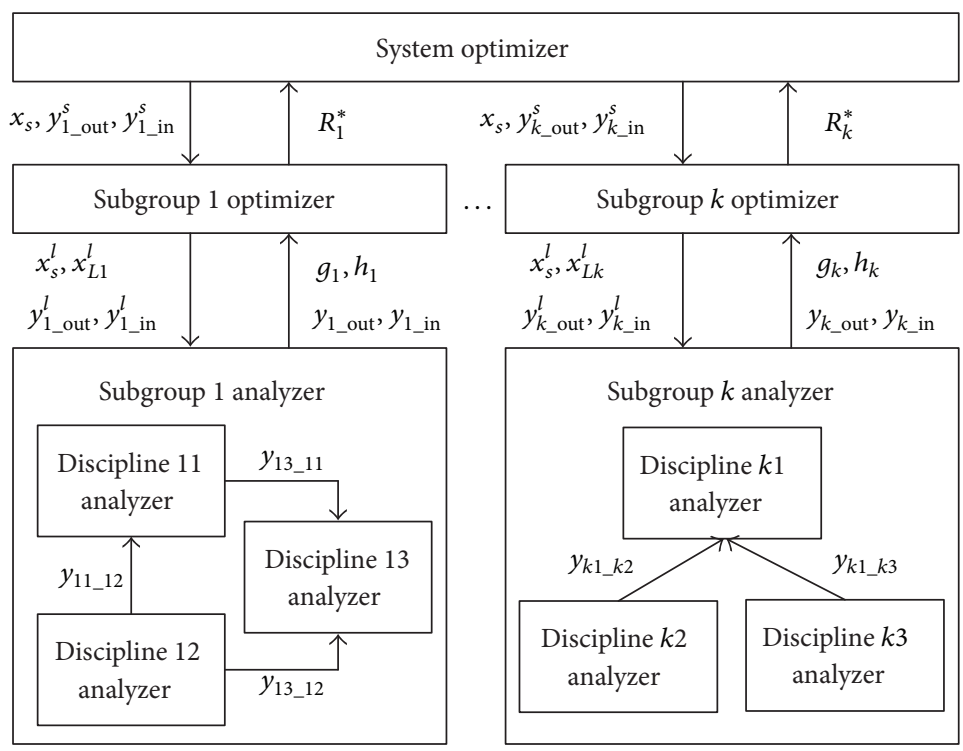

FIgURE 6: The SCO framework.

arrows from disciplines $A, B, C$, and $H$ to discipline $G$ indicate that the analysis of disciplines $A, B, C$, and $H$ depends on the output of discipline $G$. The solid lines represent the reserved disciplinary correlations and the dotted lines represent the disciplinary correlation which should be removed by adding constraints. In order to make the two disciplinary groups independent of each other, after some constraints are added to remove the interactions between $A$ and $G, H$ and $A, H$ and $E$, and $F$ and $C$, parallel analysis of the two disciplinary groups can be realized. In order to make the disciplines in each disciplinary subgroup solve sequentially in accordance with the result of division operation, after some specified constraints are added to remove the coupling correlations between $G$ and $B, G$ and $C, B$ and $H$, and $A$ and $E$, subgroup 1 can be solved in the order of $D, G, B, C$, and $H$, and subgroup 2 can be solved in the order of $A, E$, and $F$, without any complex multidisciplinary analysis.

The general idea of this study is the pretreatment of interactions between disciplines for complex multidisciplinary problems based on disciplinary DSM. Through matrix clustering algorithm, multidisciplines are divided into different disciplinary subgroups, which are sorted further by matrix division operation. A directed graph of disciplinary dependency is derived from the clustering and division results. Disciplinary interactions between subgroups and disciplinary couplings within the same group are disconnected by applying specified constraints, meeting the application demand for the sequenced collaborative optimization (SCO), as shown in Figure 6. The system layer and disciplinary subgroups keep in touch by data exchange. Disciplinary subgroups are independent of each other and each subgroup has an analyzer and an optimizer. Disciplinary analysis can be performed sequentially in each subgroup because of the pretreatment of the interactions between disciplines. This strategy improves the optimization efficiency. At the system level, the optimization target is the original objective function, and the optimization variables are constituted by global design variable $x_{s}$, coupling variables among subgroups $y_{k_{-} \text {out }}^{s}$, and coupling variables within each subgroup $y_{k_{-} \text {in }}^{s}$. System constraints are constituted by the optimal values $R_{k}^{*}$ from each subgroup used to coordinate the consistency of the coupling variables between the system and subgroups. At subgroup level, optimization variables are constituted by system variables $x_{s}^{l}$ that the subgroups need, local design variables $x_{k}^{l}$, and the coupling variables $y_{k_{\text {_in }}}^{l}$ and $y_{k_{-} \text {out }}^{l}$ within and among subgroups. The optimization target is to minimize the deviations between $x_{s}^{l}, y_{k_{\text {_in }}}^{l}$, and $y_{k_{-} \text {out }}^{l}$ and their responses passed down from the system level. Optimal values $x_{s}^{l}, x_{k}^{l}$, $y_{k_{\text {in }}}^{l}$, and $y_{k_{\text {_out }}}^{l}$ are inputs to the analyzer by the subgroup optimizer, whereas the values of equality constraints $h_{k}$, the values of inequality constraints $g_{k}$, and the coupling values $y_{k_{-} \text {out }}$ and $y_{k_{-} \text {in }}$ are returned as outputs. Although there still exist a large number of disciplinary correlations within the subgroups as Figure 6 shows, the association within the subgroups is an unidirectional tree structure because of the pretreatment of interactions between disciplines. Therefore, the analyzer in the subgroups can conduct the disciplinary analysis sequentially as the division order.

The optimization framework of SCO overcomes some drawbacks of traditional MDO strategies. It is convenient for designers to understand the complex problems by the pretreatment of interactions between disciplines based on DSM before optimization. Compared with the multidisciplinary feasible (MDF) method, although SCO adds some constraints to design space, it does not require complex multidisciplinary analysis in each subgroup. And the decoupling process belongs to the pretreatment process of the optimization, improving the solving efficiency. This improvement effect is more obvious when the disciplines couple complexly. Compared with the individual discipline feasible (IDF) method, SCO still retains a number of disciplinary correlations and removes only part of coupling interactions by adding specific constraints pertinently. It maintains the invariance of the 
design space as much as possible and can get more satisfied optimal results. Compared with all-at-once (AAO) method, SCO does not increase the design dimensions of the original optimization problem excessively. Compared with the collaborative optimization (CO) method, SCO no longer treats a single discipline as an object but classifies closely linked disciplines into a group, greatly increasing the flexibility of parallel optimization.

\section{Illustrative Example}

In order to verify the feasibility of the proposed SCO in this study and compare it with AAO method, a mathematical model, with various disciplines and a high degree of coupling, is chosen as the optimization object. Specific expression of the model is given as follows [1]:

$$
\begin{array}{ll}
\min & f \\
= & x_{2}^{2}+x_{3}+y_{1}+e^{-y_{2}}+x_{5}-0.5 x_{8}+0.8 y_{3} \\
& -2.5 y_{4}+y_{6}^{2} \\
\text { s.t. } \quad g_{1}=\frac{y_{1}}{2}-1 \geq 0, \\
g_{2}=\frac{y_{2}}{30}-1 \leq 0 \\
-10 \leq x_{1} \leq 100 \\
-10 \leq x_{2} \leq 100 \\
-10 \leq x_{3} \leq 100 \\
-10 \leq x_{4} \leq 100 \\
-10 \leq x_{5} \leq 100 \\
-10 \leq x_{6} \leq 100 \\
-10 \leq x_{7} \leq 100 \\
-10 \leq x_{8} \leq 100
\end{array}
$$

where

$$
\begin{aligned}
& y_{1}=x_{1}^{2}+x_{2}+x_{3}-0.2 y_{2}, \\
& y_{2}=\sqrt{y_{1}}+x_{1}+x_{3}, \\
& y_{3}=x_{1}+x_{3}^{3}+x_{4}-0.5 y_{1}+2 y_{5}, \\
& y_{4}=x_{3}-x_{5}+0.5 x_{6}+y_{1}^{2}-y_{3}, \\
& y_{5}=x_{1}+x_{3}-x_{7}+\sqrt{y_{2}}+2.5 y_{3}+y_{7}, \\
& y_{6}=x_{1}^{2}-y_{4}^{2}+y_{8}, \\
& y_{7}=x_{1}-x_{3}+y_{1}+0.2 y_{6}-3 y_{9}, \\
& y_{8}=x_{1}+x_{3}-x_{8}+1.5 y_{2}+\sqrt{y_{5}}, \\
& y_{9}=x_{1}+y_{4} .
\end{aligned}
$$

\begin{tabular}{llllllllll}
\hline Discipline & 1 & 2 & 3 & 4 & 5 & 6 & 7 & 8 & 9 \\
\hline 1 & 1 & 1 & 0 & 0 & 0 & 0 & 0 & 0 & 0 \\
2 & 1 & 2 & 0 & 0 & 0 & 0 & 0 & 0 & 0 \\
3 & 1 & 0 & 3 & 0 & 1 & 0 & 0 & 0 & 0 \\
4 & 1 & 0 & 1 & 4 & 0 & 0 & 0 & 0 & 0 \\
5 & 0 & 1 & 1 & 0 & 5 & 0 & 1 & 0 & 0 \\
6 & 0 & 0 & 0 & 1 & 0 & 6 & 0 & 1 & 0 \\
7 & 1 & 0 & 0 & 0 & 0 & 1 & 7 & 0 & 1 \\
8 & 0 & 1 & 0 & 0 & 1 & 0 & 0 & 8 & 0 \\
9 & 0 & 0 & 0 & 1 & 0 & 0 & 0 & 0 & 9 \\
\hline
\end{tabular}

FIGURE 7: The DSM of disciplines.

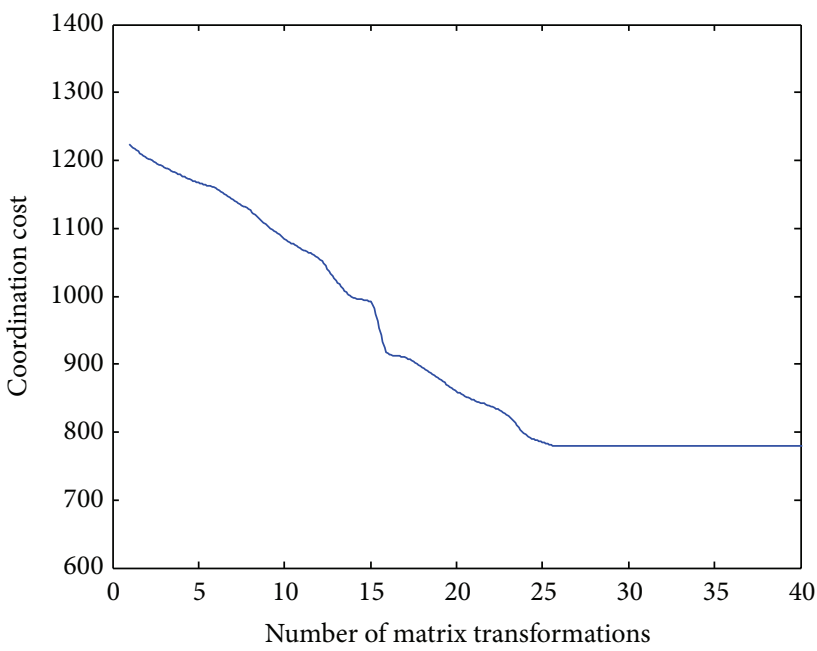

FIgURE 8: The curve of coordination cost.

The optimization problem consists of 8 design variables and 9 mutually coupling disciplines. The interactions between the disciplines are clearly shown in Figure 7, with a total of 17 correlations.

After the clustering analysis of the disciplinary interaction matrix, the nine disciplines are divided into two subgroups, namely, $1,2,3,5,7$, and 8 in one subgroup and 4, 6, and 9 in the other one. The curve of coordination cost and the clustered result are, respectively, shown in Figures 8 and 9. The most correlation relationships between disciplines gather within subgroups.

Figure 10 shows the result after the partitioning operation on the two clustering subgroups shown in Figure 9.

There are still some disciplinary correlations between the subgroups and some coupling interactions within each subgroup after the clustering and division operations. The directed graph for decoupling as shown in Figure 11, can be derived from the clustering and division result in Figure 10. In order to meet the requirement of the SCO framework, some constraints are added to make two subgroups independent of each other and remove the coupling relations within subgroups, for example the dependencies from discipline 1 
TABLE 1: The optimized result.

(a)

\begin{tabular}{lccccccc}
\hline \multirow{2}{*}{ Method } & \multicolumn{9}{c}{ The optimized location } & $x_{5}$ & $x_{6}$ & $x_{7}$ \\
& $x_{1}$ & $x_{2}$ & $x_{3}$ & $x_{4}$ & $x_{5}$ & -2.9131 \\
\hline SCO & -1.3959 & 0.0677 & -0.0187 & -9.7973 & -10 & -10 & -6.7511 \\
AAO & -1.2029 & 0.0830 & 0.6403 & -10 & -10 & -10 & 1.0927 \\
\hline
\end{tabular}

(b)

\begin{tabular}{|c|c|c|c|c|c|c|c|c|c|c|}
\hline \multirow{2}{*}{ Method } & \multicolumn{9}{|c|}{ The values of coupling variables } & \multirow{2}{*}{ Target $f$} \\
\hline & $y_{1}$ & $y_{2}$ & $y_{3}$ & $y_{4}$ & $y_{5}$ & $y_{6}$ & $y_{7}$ & $y_{8}$ & $y_{9}$ & \\
\hline SCO & 2 & 0.0028 & 4.7440 & 3.2341 & 8.4686 & -0.2540 & -4.9452 & 8.2539 & 1.8393 & -7.8670 \\
\hline $\mathrm{AAO}$ & 2 & 0.8516 & 6.3816 & 3.2587 & 9.1610 & -0.2500 & -6.0606 & 8.9221 & 2.0558 & -7.3147 \\
\hline
\end{tabular}

\begin{tabular}{llllllllll}
\hline Discipline & 1 & 2 & 3 & 5 & 7 & 8 & 4 & 6 & 9 \\
\hline 1 & 1 & 1 & 0 & 0 & 0 & 0 & 0 & 0 & 0 \\
2 & 1 & 2 & 0 & 0 & 0 & 0 & 0 & 0 & 0 \\
3 & 1 & 0 & 3 & 1 & 0 & 0 & 0 & 0 & 0 \\
5 & 0 & 1 & 1 & 5 & 1 & 0 & 0 & 0 & 0 \\
7 & 1 & 0 & 0 & 0 & 7 & 0 & 0 & 1 & 1 \\
8 & 0 & 1 & 0 & 1 & 0 & 8 & 0 & 0 & 0 \\
4 & 1 & 0 & 1 & 0 & 0 & 0 & 4 & 0 & 0 \\
6 & 0 & 0 & 0 & 0 & 0 & 1 & 1 & 6 & 0 \\
9 & 0 & 0 & 0 & 0 & 0 & 0 & 1 & 0 & 9 \\
\hline
\end{tabular}

Figure 9: The clustered result.

\begin{tabular}{llllllllll}
\hline Discipline & 1 & 2 & 7 & 3 & 5 & 8 & 4 & 6 & 9 \\
\hline 1 & 1 & 1 & 0 & 0 & 0 & 0 & 0 & 0 & 0 \\
2 & 1 & 2 & 0 & 0 & 0 & 0 & 0 & 0 & 0 \\
7 & 1 & 0 & 7 & 0 & 0 & 0 & 0 & 1 & 1 \\
3 & 1 & 0 & 0 & 3 & 1 & 0 & 0 & 0 & 0 \\
5 & 0 & 1 & 1 & 1 & 5 & 0 & 0 & 0 & 0 \\
8 & 0 & 1 & 0 & 0 & 1 & 8 & 0 & 0 & 0 \\
4 & 1 & 0 & 0 & 1 & 0 & 0 & 4 & 0 & 0 \\
6 & 0 & 0 & 0 & 0 & 0 & 1 & 1 & 6 & 0 \\
9 & 0 & 0 & 0 & 0 & 0 & 0 & 1 & 0 & 9 \\
\hline
\end{tabular}

FIgURE 10: The partitioned result.

to discipline 2, discipline 3 to discipline 5 , and the dependency between the two subgroups. The solid lines represent the reserved disciplinary correlations and the dotted lines represent the disciplinary correlations which should be removed. The figure still retains many disciplinary dependencies because these dependencies do not form any coupling

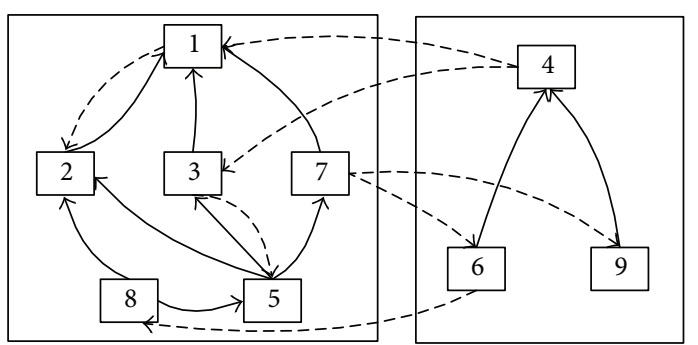

FIGURE 11: The directed graph for decoupling.

association and will not affect the parallel optimization in the disciplinary subgroups. The output values of respective disciplines can be got after an analysis according to the discipline sequence obtained by the division.

\section{Result}

Table 1 shows the optimized results obtained using AAO and SCO strategies. The target value obtained from SCO (i.e., -7.8670 ) is better than that from $\mathrm{AAO}$ (i.e., -7.3147), verifying the validity of the proposed SCO in this paper. AAO method converts state variables into independent optimization variables in the optimization process, which increases the scale of the original problem and makes the solving process harder. SCO method converts the original optimization problem into multiple subgroups of parallel analysis through the clustering analysis of the disciplinary matrix. The disciplinary analysis in each subgroup can be executed sequentially after the division and decoupling operations, which reduces the solving difficulty. It can be seen from the optimized result that SCO has a better optimization effect.

\section{Conclusion}

This study uses DSM to describe disciplinary interactions for MDO problems, introduces two parameters, that is, bid values and coordination cost values, to achieve clustering algorithm for the disciplinary interaction matrix, and aggregates those mutually coupling disciplines into the groups with strong ties among similar ones and weak ties among heterogeneous ones. Further, those in the same group are 
sequenced by the division algorithm, and the number of disciplinary feedback correlations is reduced. A directed graph of disciplinary dependency is derived from the clustering and division results. By adding constraints, the groups are made independent and a tree structure with no coupling relation within the same subgroup is obtained, thus forming MDO approach enhanced with DSM algorithms, that is, a socalled sequenced collaborative optimization (SCO). Not only is SCO theoretically addressed but also a concrete example is used to compare SCO and AAO.

Although the preliminary study suggests that SCO optimization framework works well in solving MDO problems, the illustrated example is not very complex. In future work, more complex optimization problems and models in the real world will be used to verify the solving process of the SCO framework through approximate modeling technology as well as clustering and division algorithms of DSM to deal with the relationships between disciplines.

\section{Competing Interests}

The authors declare that there is no conflict of interests regarding the publication of this paper.

\section{Acknowledgments}

The project was supported by the State Key Laboratory of Automotive Simulation and Control Open Foundation (20121112), the National Natural Science Foundation of China (51175187 and 51375168), the Science and Technology Foundation of Guangdong Province (2014A020223003, 2015A020220004, 2016B090918035, and 2016A020228005), and the Science and Technology Foundation of Zhanjiang City (2015A01001).

\section{References}

[1] W. Q. Yuan, Y. S. Liu, X. P. Ye, and D. H. Liu, "A multidisciplinary approach combining with topological decoupling," Journal of Computer-Aided Design \& Computer Graphics, vol. 27, no. 9, pp. 1795-1804, 2015.

[2] C. Bakker, G. T. Parks, and J. P. Jarrett, "Optimization algorithms and ODE's in MDO," in Proceedings of the ASME International Design Engineering Technical Conferences and Computers and Information in Engineering Conference (IDETC/CIE '13), vol. 36, 10 pages, ASME, Portland, Ore, USA, August 2013.

[3] R. Q. Wang, Z. G. Jia, D. Y. Hu, and H. Jin, "Multiple precision MDO strategy for turbine blade," Journal of Aerospace Power, vol. 28, no. 5, pp. 961-970, 2013.

[4] I. Ferreira, J. A. Cabral, P. Saraiva, and M. C. Oliveira, "A multidisciplinary framework to support the design of injection mold tools," Structural \& Multidisciplinary Optimization, vol. 49, no. 3, pp. 501-521, 2014.

[5] H. Su, L.-X. Gu, and C.-L. Gong, "Universal multidisciplinary optimization architecture based on disciplinary relation matrix," Computer Integrated Manufacturing Systems, vol. 20, no. 4, pp. 731-738, 2014.

[6] J. S. Robinson, "An overview of NASA's integrated design and engineering analysis (IDEA) environment," in Proceedings of the 17th AIAA International Space Planes and Hypersonic Systems and Technologies Conference, San Francisco, Calif, USA, April 2011.

[7] J. Gray, K. T. Moore, T. A. Hearn, and B. A. Naylor, "Standard platform for benchmarking multidisciplinary design analysis and optimization architectures," AIAA Journal, vol. 51, no. 10, pp. 2380-2394, 2013.

[8] T. L. Kenworthy and C. G. Jensen, "Cad-centric dynamic workflow generation," Computer-Aided Design and Applications, vol. 6, no. 5, pp. 673-683, 2009.

[9] K. Meruva, Z. Bi, D. Mueller, and B. Kang, "Formulation and validation of multidisciplinary design problem on wear and fatigue life of lead screw actuators," Mathematical Problems in Engineering, vol. 2013, Article ID 303967, 10 pages, 2013.

[10] Q. Cheng, C. Zhan, Z. Liu, Y. Zhao, and P. Gu, "Sensitivitybased multidisciplinary optimal design of a hydrostatic rotary table with particle swarm optimization," Journal of Mechanical Engineering, vol. 61, no. 7-8, pp. 432-447, 2015.

[11] R. Y. Su, L. J. Gui, Z. B. Wu, C. Tian, L. Ma, and Z. J. Fan, "Multidisciplinary design and collaborative optimization for bus body," Journal of Mechanical Engineering, vol. 46, no. 18, pp. 128-133, 2010.

[12] P. Jiang, J. Wang, Q. Zhou, and X. Zhang, "An enhanced analytical target cascading and Kriging model combined approach for multidisciplinary design optimization," Mathematical Problems in Engineering, vol. 2015, Article ID 685958, 11 pages, 2015.

[13] D. V. Steward, "Design structure system: a method for managing the design of complex systems," IEEE Transactions on Engineering Management, vol. 28, no. 3, pp. 71-74, 1981.

[14] T. Habib, "Multidisciplinary product decomposition and analysis based on design structure matrix modeling," in Proceedings of the 7th World Conference on Mass Customization, Personalization, and Co-Creation (MCPC '14), pp. 409-423, Aalborg, Denmark, February 2014.

[15] A. B. Lambe and J. R. R. A. Martins, "Extensions to the design structure matrix for the description of multidisciplinary design, analysis, and optimization processes," Structural and Multidisciplinary Optimization, vol. 46, no. 2, pp. 273-284, 2012.

[16] X.-Q. Yan, Y. Li, W.-Q. Li, and Y.-J. Wan, "Decoupling planning method of MDO based on coupling strength and FDSM," Computer Integrated Manufacturing Systems, vol. 19, no. 7, pp. 1447-1455, 2013.

[17] R. E. Thebeau, Knowledge management of system interfaces and interactions for product development processes [M.S. thesis], MIT, Cambridge, Mass, USA, 2001.

[18] T. Chen and R. Xiao, "A dynamic intelligent decision approach to dependency modeling of project tasks in complex engineering system optimization," Mathematical Problems in Engineering, vol. 2013, Article ID 398123, 12 pages, 2013. 


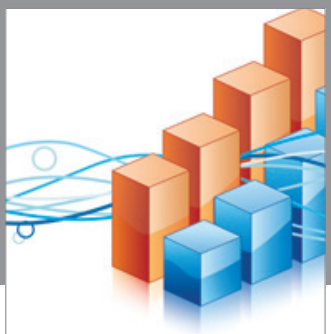

Advances in

Operations Research

vatem alat4

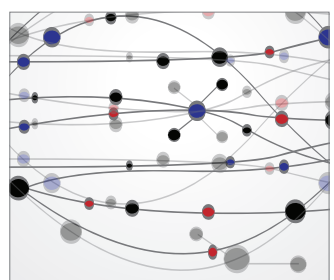

\section{The Scientific} World Journal
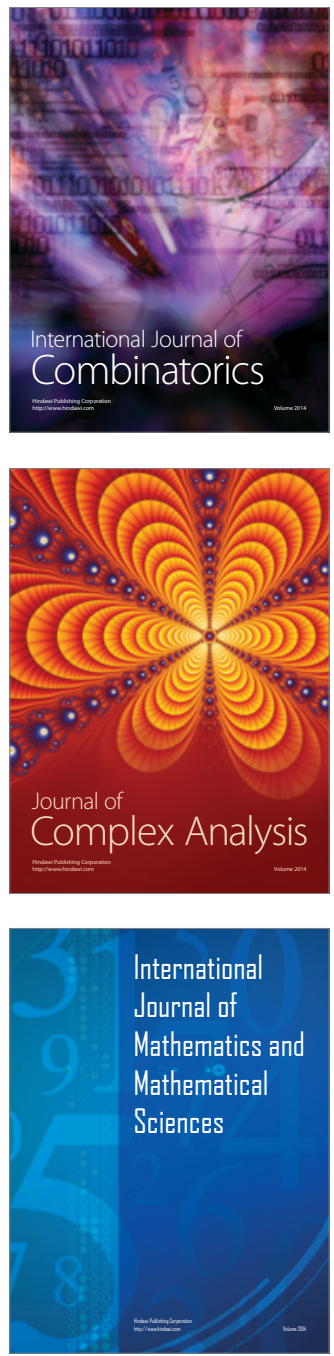
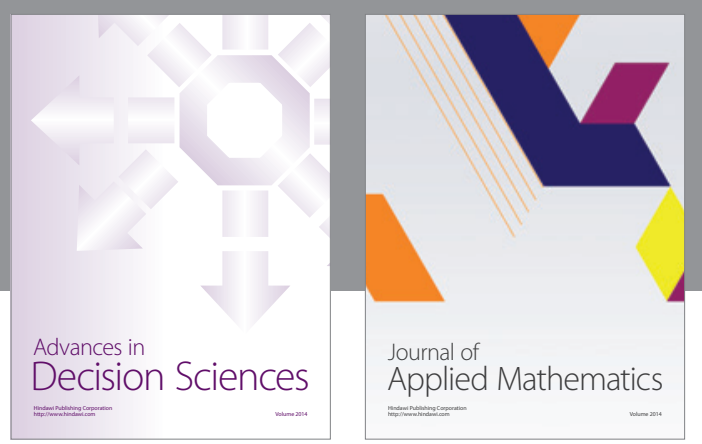

Algebra

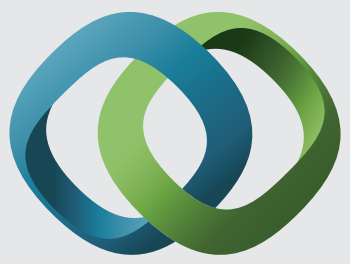

\section{Hindawi}

Submit your manuscripts at

http://www.hindawi.com
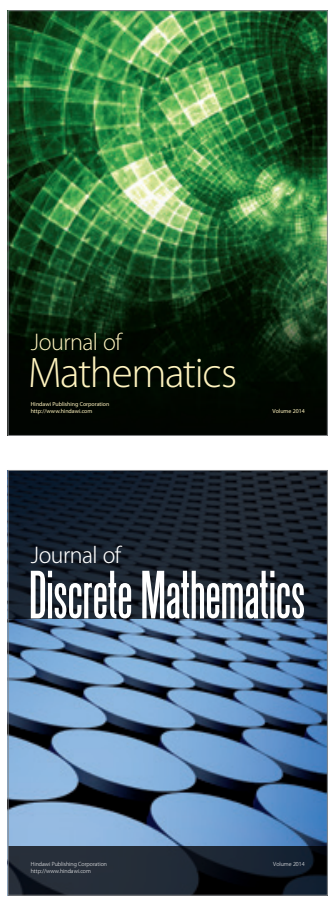

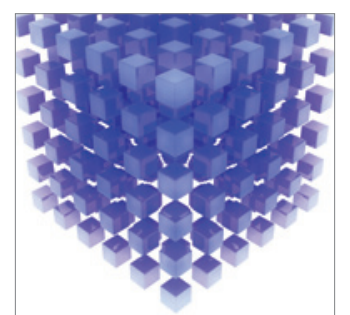

Mathematical Problems in Engineering
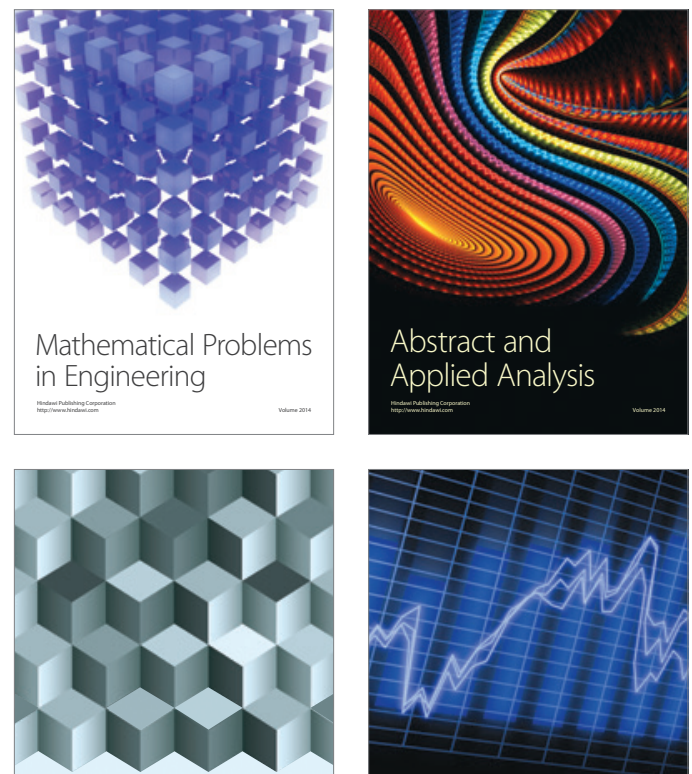

Journal of

Function Spaces

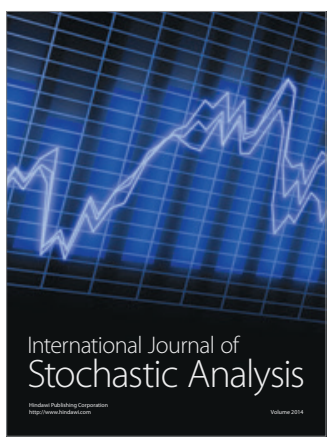

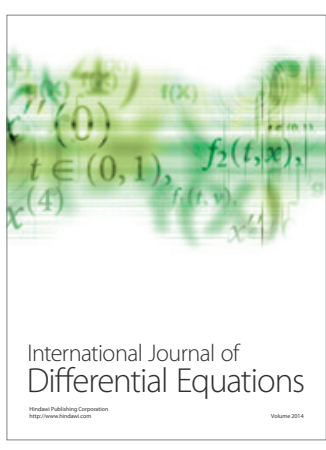
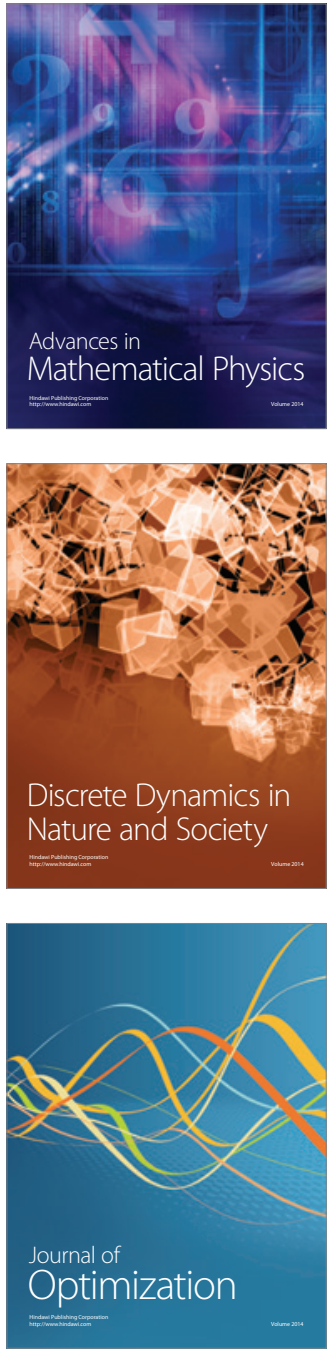